\title{
REVIEW \\ Posttraumatic stress following spinal cord injury: a systematic review of risk and vulnerability factors
}

\author{
K Pollock ${ }^{1,3}$, D Dorstyn ${ }^{1,3}$, L Butt ${ }^{2}$ and S Prentice ${ }^{1}$
}

Objectives: To summarise quantitatively the available evidence relating to pretraumatic, peritraumatic and posttraumatic characteristics that may increase or decrease the risk of developing posttraumatic stress disorder (PTSD) following spinal cord injury $(\mathrm{SCl})$.

Study design: Systematic review.

Methods: Seventeen studies were identified from the PubMed, Psyclnfo, Embase, Scopus, CINAHL, Web of Science and PILOTS databases. Effect size estimates ( $r$ ) with associated $95 \%$ confidence intervals (Cls), $P$-values and fail-safe $N$ s were calculated.

Results: Individual studies reported medium-to-large associations between factors that occurred before (psychiatric history $r=0.48$ (95\% Cl, 0.23-0.79) $P=0.01$ ) or at the time of injury (tetraplegia $r=-0.36(95 \% \mathrm{Cl},-0.50$ to -0.19$) P<0.01$ ). Postinjury factors had the strongest pooled effects: depressed mood $\left(r_{\mathrm{w}}=0.64,(95 \% \mathrm{Cl}, 0.54-0.72)\right)$, negative appraisals $\left(r_{\mathrm{w}}=0.63(95 \% \mathrm{Cl}, 0.52-\right.$ $0.72))$, distress $\left(r_{\mathrm{w}}=0.57(95 \% \mathrm{Cl}, 0.50-0.62)\right)$, anxiety $\left(r_{w}=0.56(95 \% \mathrm{Cl}, 0.49-0.61)\right)$ and pain severity $\left(r_{\mathrm{w}}=0.35(95 \% \mathrm{Cl}, 0.27-\right.$ $0.43)$ ) were consistently related to worsening PTSD symptoms $(P<0.01)$. Level of injury significantly correlated with current PTSD severity for veteran populations $\left(Q_{\mathrm{B}}(1)=18.25, P<0.001\right)$, although this was based on limited data.

Conclusion: Combinations of peri- and post-injury factors appear to be influential in the development of PTSD among persons with $\mathrm{SCl}$. Further studies are needed to extrapolate these findings to the broader spinal cord-injured population. More longitudinal research, driven by multicausal models of causation such as the diathesis-stress model, is also needed to determine the temporality of PTSD risk factors.

Spinal Cord (2017) 55, 800-811; doi:10.1038/sc.2017.45; published online 9 May 2017

\section{INTRODUCTION}

Traumatic stress reactions are common following an acquired spinal cord injury (SCI), with $40 \%$ of adults reporting an acute symptom pattern of avoidance, heightened (hyper) anxiety and intrusive trauma memories. ${ }^{1}$ In total, $4 \%$ experience these symptoms beyond 1 month after SCI, warranting a diagnosis of posttraumatic stress disorder (PTSD). ${ }^{2}$ Without treatment, the lifetime prevalence of PTSD for those with SCI remains high: up to $29 \%$ continue to report symptoms 30 years after injury. ${ }^{1}$ Consistent with a diathesis-stress conceptualisation, a complex interplay of individual and contextual factors present before (pre), during (peri) or post-SCI appears to increase vulnerability to the development of PTSD. ${ }^{1,3,4}$ Knowledge of these factors is important in order to accurately identify and support high-risk individuals. A better understanding of the factors that may exacerbate partial (subsyndromal or acute) PTSD can also help clinicians provide the necessary treatment before symptoms become chronic.

Female gender, in particular, may place injured adults at risk of developing PTSD. ${ }^{4}$ Indeed, gender is a salient risk factor for other post-injury negative emotional responses, including depression, ${ }^{5,6}$ despite a greater injury incidence and prevalence among males (80\% males: $20 \%$ females). ${ }^{7}$ However, gender may influence PTSD only to the extent that other sociocontextual factors are present. This includes lower levels of education and income and being divorced or widowed-although the identified connections between educational attainment, family stability and distress vary in magnitude., ${ }^{2,-11}$ The likelihood that an individual with SCI will develop PTSD after injury may also be determined by preexisting mental health problems (for example, depression, anxiety), negative coping styles and prior exposure to trauma. ${ }^{2,5,8,12,13}$

There are few data in relation to the role that peritrauma factors, namely SCI characteristics, have in PTSD aetiology and maintenance. Regardless of cause, a spinal cord lesion can be viewed as a traumatic event, with life-threatening SCI complications (for example, cardiovascular, respiratory) potentially reinforcing an elevated fear of death-considered a fundamental source of PTSD. ${ }^{14}$ It is also postulated that individuals with higher level of injury experience more severe emotional reactions, resulting from a dependence on others for day-to-day tasks. ${ }^{15,16}$ From a neurobiological perspective, sensory and motor pathways are compromised following tetraplegic injury which, in turn, may impede PTSD symptoms of sympathetic arousal (for example, hypervigilance, exaggerated startle response). ${ }^{17}$ However, anecdotal clinical lines of evidence suggest that those with long-term paraplegia may struggle more so. This may relate to a sense that their degree of impairment, which commonly includes 'invisible'

${ }^{1}$ School of Psychology, Faculty of Health and Medical Sciences, University of Adelaide, Adelaide, South Australia, Australia and ${ }^{2}$ Craig Hospital, Englewood, CO, USA

${ }^{3}$ These authors contributed equally to this work.

Correspondence: Dr D Dorstyn, School of Psychology, Faculty of Health and Medical Sciences, University of Adelaide, North Terrace Campus, Adelaide, South Australia 5005, Australia.

E-mail: diana.dorstyn@adelaide.edu.au

Received 8 January 2017; revised 22 March 2017; accepted 23 March 2017; published online 9 May 2017 
impairments such as chronic pain and incontinence, does not neatly fit the stereotypical view of disability. ${ }^{18}$ Further support for these theories is, however, needed. In addition, veteran studies have demonstrated that intoxication or substance abuse at the time of injury can be detrimental to mental health outcomes. ${ }^{17,19}$ The generalisability of these findings to the broader SCI population is, however, limited given that this subgroup may be at greater risk of mental illness due to their experience of prior trauma. ${ }^{20,21}$

Perhaps the most consistent evidence relates to the role of post-SCI complications and comorbidities and risk of PTSD. It is thought that chronic neuropathic or nociceptive pain and psychological distress are mutually maintaining. ${ }^{22,23}$ Reactions of depression and PTSD also commonly co-occur. ${ }^{5,6,24}$ Both depression and PTSD impede disability acceptance by reinforcing negative cognitions about the self (including 'self-blame') and the world. ${ }^{12}$ Notably, this research is characterised by self-reported screening measures of trauma impact, which vary in the degree to which they map onto standardised diagnostic nomenclature (for example, Diagnostic and Statistical Manual of Psychiatric Disorder (DSM)). However, few SCI studies have empirically tested the impact of PTSD measurement type on risk factor estimates.

In summary, the burden of PTSD symptoms in those with SCI has been described. This provides an opportunity to quantitatively summarise the data, which, to date, have not occurred. Our objective was to evaluate a range of pre-, peri- and post-SCI factors that can potentially lead to PTSD by performing a systematic and meta-analytic review of the available research. A further aim was to examine the differential effects of sample (that is, military veterans) and measurement (that is, dichotomous, diagnostic measures versus continuous, symptom measures) characteristics on these findings. Research gaps in the current evidence base were also identified. ${ }^{25}$

\section{METHODS}

\section{Literature search}

A search of seven electronic databases (PubMed, PsycINFO, Web of Science, Scopus, Embase, CINAHL, PILOTS) was conducted for the period between January 1980 (to coincide with the inclusion of PTSD as a diagnostic category in the DSM-III) and December 2016. Search terms were tailored to each database and included a broad list of keywords and phrases related to SCI (for example, spine, spinal, spinal cord trauma, spinal cord fracture) and posttraumatic stress (for example, PTSD, traumatic neurosis, shell shock, war neurosis and combat stress) (see Appendix for logic grids with Boolean operators). A research librarian assisted with the development of the search terms to ensure their accuracy. The reference lists of all included studies were searched, as were book chapters ${ }^{26}$ and relevant reviews, ${ }^{1,27-33}$ in order to identify any research that may have been missed. A request for articles in press was additionally made through the American Psychological Association's Division 22 (Rehabilitation Psychology) Listserv. As a countercheck, international peer-reviewed journals targeted to SCI rehabilitation (Archives of Physical Medicine and Rehabilitation, Disability and Rehabilitation, European Spine Journal, Journal of Spinal Cord Medicine, SCI Nursing, Spine, Spinal Cord, Rehabilitation Psychology, The Spine Journal, Topics in Spinal Cord Injury Rehabilitation) were electronically searched using 'PTSD' and 'post-traumatic stress' as keywords.

\section{Eligibility criteria}

Studies had to meet the following criteria for inclusion in this review: (1) participants with an acquired (traumatic or nontraumatic) SCI were assessed as a young child (that is, 8-11 years), adolescent (12-17 years) or adult (aged 18+ years); and (2) current PTSD symptomatology was assessed using a standardised self-report or clinician-based instrument. This included a broad group of participants: individuals experiencing acute stress symptoms and those reporting re-experiencing, avoidance and arousal symptoms consistent with established (DSM) PTSD criteria. (3) Studies also had to assess risk factors for PTSD as primary or secondary outcomes. This included pre-injury (for example, age, gender), injury-specific (for example, SCI level) and post-injury variables (that is, SCI sequelae). A risk factor was defined as any variable thought to contribute to symptom severity or diagnostic status. ${ }^{31-33}$ (4) Studies also had to provide parametric data to enable the calculation of an effect size $r$. This included studies using a group design (which reported $t$-tests, chi-square $\chi^{2}$ and exact $P$-values) or a correlational design (reporting Pearson's or point-biserial $r$ ). (5) Finally, studies had to be published in a journal in English. ${ }^{34}$ To ensure generalisability of the findings, only PTSD correlates examined by two or more studies were considered. ${ }^{35}$ Studies that examined multiple trauma groups or those that included individuals with spinal cord disorders of congenital or disease origin, without appropriate disaggregation within the results, were excluded. Additionally, studies that only provided data from multivariate analysis (for example, regression, factor analysis, structural equation modelling) were ineligible. ${ }^{36}$

Reliability of the article selection process was checked, with a second (DD) and third reviewer (SP) screening the titles and abstracts of 20 eligible articles randomly selected by the primary reviewer (KP). Inter-rater agreement was moderate $(\kappa=0.60$ (95\% confidence interval (CI), 0.17-0.97)). Discrepancies, which focussed on studies to exclude, were discussed and resolved by consensus.

Of the initial 1307 articles, 30 eligible studies were identified. These studies were further examined to ensure independence of the samples..$^{36}$ Lead authors of studies were contacted for clarification where necessary. ${ }^{5,14,15}$ Sample overlap was identified in 20 studies led or co-authored by Boyer, ${ }^{37,38}$ Chung $^{13,39,40}$ Kennedy, ${ }^{41,42}$ Martz, ${ }^{14,43,44}$ Livneh, $^{5,45}$ Nielsen ${ }^{10,15}$ and Radnitz. ${ }^{8,9,17,21,46,47}$ These studies were subsequently combined and treated as seven independent studies. Where a single study contributed multiple effect estimates for the same risk factor, data from the largest sample or most recently published article were used to ensure no duplication. This resulted in a final sample of 17 independent studies (see Figure 1).

\section{Risk of bias}

The methodological quality of included studies was assessed using a checklist modelled from existing rating tools. ${ }^{48-51}$ Studies were rated on components that are considered critical to clinical research: statistical power (whether the study findings could be attributed to chance); internal validity (degree to which a study minimises bias in measurement and data collection) and external validity (extent to which the study findings can be generalised to the broader SCI population). ${ }^{52}$ Two reviewers (KP and DD) were involved in the rating process, with each independently evaluating each study. Inter-rater reliability was high for all judgements (agreement $=94 \%$ ).

\section{Data collection and preparation}

In line with evidence-based recommendations for the reporting of meta-analyses, ${ }^{53}$ a data extraction sheet was developed to summarise key information from each study. Extracted data included the following: (1) sample demographics (for example, mean age, gender); (2) injury characteristics (for example, SCI severity, duration); (3) effect-size data (for example, means, s.d., correlations, $t$-tests, chi-square $\chi^{2}$ ); (4) study characteristics (for example, PTSD measure, recruitment source); and (5) risk factors. Eighteen risk factors were identified and classified into one of the following three broad categories: pre-SCI (that is, gender, age at injury, relationship status, education, psychiatric history and previous trauma), peri-SCI (that is, lesion completeness, SCI level, age at injury, alcohol/substance use at injury and loss of consciousness); and post-SCI (that is, time since SCI, pain level, posttraumatic cognitions, comorbid depression, anxiety, distress and social support).

\section{Data analysis}

The correlation coefficient $r$, which quantifies the strength and direction of a relationship between two dichotomous or continuous variables, was selected as the primary effect size for review as it was the most commonly reported metric by eligible studies. Studies that did not directly report $r$ provided test statistics $\left(\chi^{2}, t\right.$ or $\mathrm{F}$, exact $P$-value, frequency count), which can be converted. ${ }^{54,55}$ To ensure that all effect sizes reflected the same relationship direction, lead authors were contacted to clarify the coding of their risk factors. ${ }^{37,41,43}$ 


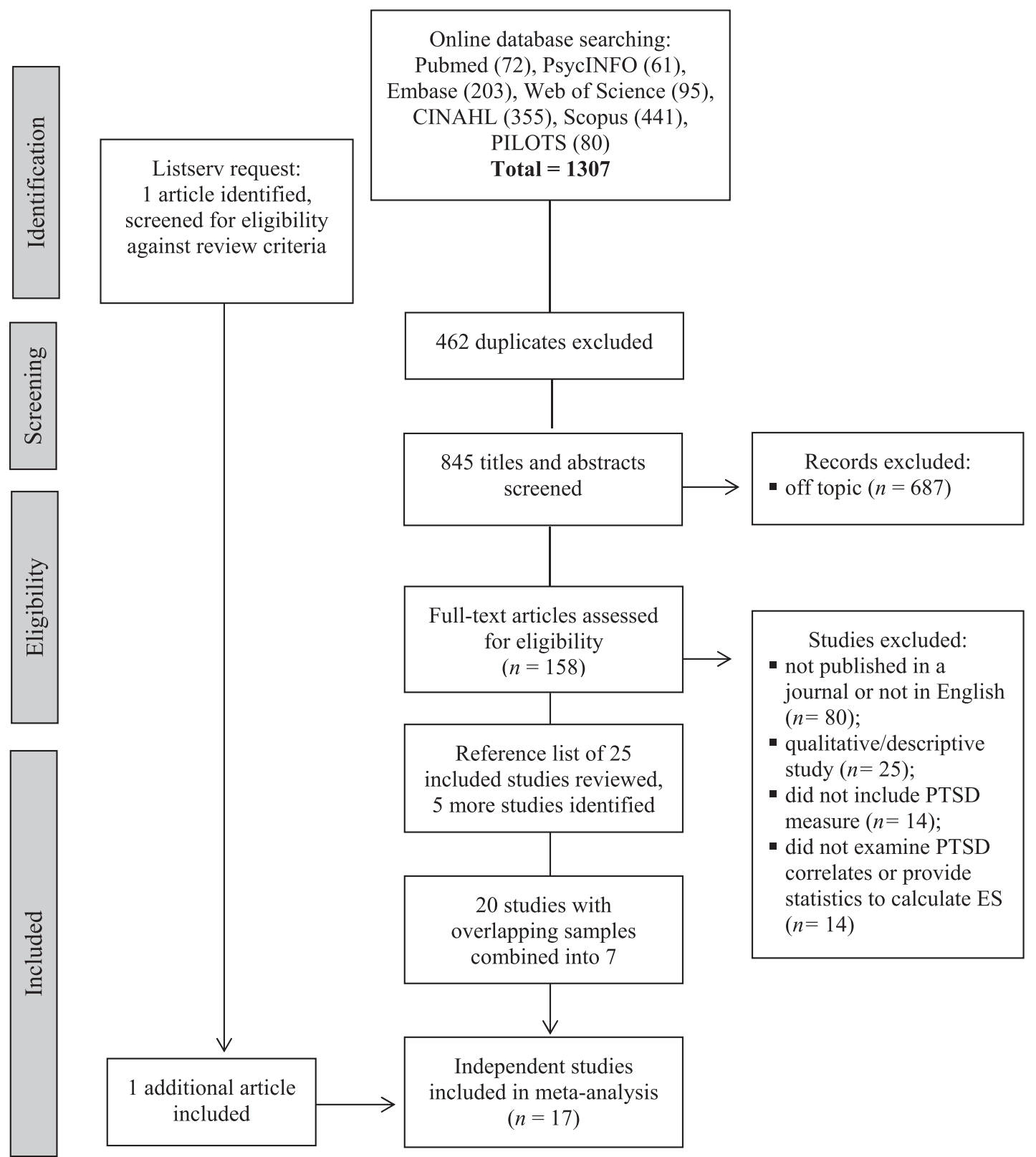

Figure 1 PRISMA flowchart of study selection process.

Effect sizes were interpreted as per Cohen's guidelines, ${ }^{56}$ with correlations of $0.10,0.30$ and 0.50 representing small, medium and large associations, respectively.

The calculation of effect sizes was a multistage process. First, an individual $r$ was obtained for each relationship between a standardised measure of PTSD and risk factor. Second, if a study provided multiple-effect sizes for a single risk factor (for example, several PTSD measures), an average $r$ was calculated. This involved transforming individual $r$ s to Fisher's $Z$ (so that a normal distribution could be provided), computing the average Fisher's $Z$ and then backtransforming this average to $r .{ }^{36}$ This ensured that each study only contributed a single-effect estimate to the pooled $r$ for any given risk factor. ${ }^{36}$ Third, effect sizes from different studies that assessed the same risk factor were grouped by PTSD measure and then pooled. The only exception to this was social support, where individual effect estimates were considered for this multidimensional risk factor. Similarly, effect estimates for studies that examined acute versus chronic PTSD were separately examined. As the reliability of individual effect sizes is somewhat dependent on their underlying sample size (that is, effect sizes from larger samples embody less sampling error and, therefore, are more precise in their estimate), study $r$ s were weighted by their inverse variance before being pooled (mean $r_{\mathrm{w}}$ ). ${ }^{36}$ Weighting was based on a random-effects model. ${ }^{57}$

To determine the accuracy of individual and weighted effect sizes, 95\% CIs and exact $P$-values were calculated. Effect sizes were considered statistically significant if the $\mathrm{CI}$ did not include the value of zero and the associated $P$-value was $>0.05 .{ }^{58}$ In addition, fail-safe Ns $\left(N_{\mathrm{fs}}\right)$ were calculated to address a potential validity threat-publication bias. ${ }^{59} N_{\mathrm{fs}}$ represents the hypothetical number of unpublished or unidentified studies reporting no effect (that is, no relationship) required to render a calculated effect size as meaningless (that is, $r<0.1$ ). The higher the $N_{\mathrm{fs}}$ value, the more confidence we can have in the result. A conservative approach was adopted in this meta-analysis, with an $N_{\mathrm{fs}}$ value needing to exceed the number of studies contributing to an effect-size estimate (that is, $N_{\mathrm{fs}}>N_{\text {studies }}$ ) to be considered robust.

The $I^{2}$ statistic was calculated to assess the degree of consistency in pooled effect-size estimates. ${ }^{60}$ The value of $I^{2}$ represents the percentage of betweenstudies variance that can be accounted for by actual differences as opposed to 
chance. $I^{2}$ values $>50 \%$ suggest moderate-to-substantial heterogeneity across individual effect-size estimates, potentially due to methodological variation in addition to clinical heterogeneity that characterises research with medical populations. ${ }^{25}$ Importantly, calculation of the $I^{2}$ statistic is not dependent on the number of studies included in a meta-analysis. ${ }^{60}$

The moderating effects of sample (veterans versus civilians) and measurement characteristics (self-report versus clinician rating) were additionally evaluated for those risk factors associated with statistical heterogeneity $\left(I^{2} \geqslant 50 \%\right) \cdot{ }^{60}$ Specifically, effect estimates for subsets of studies that investigated the same risk factor (for example, SCI level) were combined, once again using Fisher's $Z_{r}$ transformation and back transformation. Group mean differences were then examined using the $Q$-test of homogeneity and a mixed-effects model (which assumes some within-group variation of true effect estimates). ${ }^{57}$ These analyses were conducted with the Comprehensive Meta-Analysis Software (Version 3, 2014 Biostat, Englewood, NJ, USA).

In combination, these statistics were used to assess the clinical relevance of each potential PTSD risk factor for individuals with an acquired SCI. Specifically, a risk factor was considered an important predictor of PTSD

Table 1 Study characteristics $\left(N_{\text {studies }}=17\right)$

\begin{tabular}{|c|c|c|c|c|c|c|c|}
\hline Lead author & Country & $\begin{array}{l}N \text { (male: } \\
\text { female) }\end{array}$ & $\begin{array}{l}\text { Mean age } \\
\text { (s.d.) }\end{array}$ & $\begin{array}{l}\text { Mean time since injury } \\
\text { (s.d.) }\end{array}$ & Injury cause & Recruitment source & PTSD measure \\
\hline Agar $^{12}$ & UK & $50(43: 7)$ & $\begin{array}{l}38.9 \\
(13.42)\end{array}$ & 3-24 months post & Traumatic & Inpatient & PDS; IES \\
\hline Boyer 37,38 & USA & $21(9: 12)$ & - & 5.9 years $(4.4)$ & $\begin{array}{l}\text { Traumatic and } \\
\text { non-traumatic }\end{array}$ & Inpatient and outpatient & PDS; CPSS \\
\hline Chung $13,39,40$ & Greece & $62(43: 19)$ & $\begin{array}{l}45.08 \\
(13.97)\end{array}$ & 1.7 years $(3.46)$ & $\begin{array}{l}\text { Traumatic and } \\
\text { non-traumatic }\end{array}$ & Inpatient & PCL \\
\hline Hatcher ${ }^{4}$ & UK & $102(83: 19)$ & $\begin{array}{l}46.66 \\
(10.98)\end{array}$ & 15.2 years $(11.76)$ & non-traumatic & Inpatient and outpatient & IES \\
\hline Kennedy ${ }^{1,41,42}$ & UK & $85(68: 17)$ & $32.6(-)$ & $6-24$ weeks post & Traumatic & Inpatient & IES \\
\hline Krause $^{65}$ & USA & $\begin{array}{l}927 \\
(662: 265)\end{array}$ & - & 22.5 years $(10.7)$ & Traumatic & Outpatient & PPTSD-R \\
\hline Livneh $^{5,45}$ & USA & $95(68: 27)$ & $\begin{array}{l}47.5 \\
(17.61)\end{array}$ & 7.5 years $(6.8)$ & $\begin{array}{l}\text { Traumatic and } \\
\text { non-traumatic }\end{array}$ & Outpatient & PPTSD-R \\
\hline Martz $^{14,43,44}$ & USA & $312(271: 42)$ & $50.7(14.8)$ & 14.1 years $(13.0)$ & $\begin{array}{l}\text { Traumatic and } \\
\text { non-traumatic }\end{array}$ & Outpatient & PPTSD-R \\
\hline Migliorini 6 & Australia & $443(346: 97)$ & $\begin{array}{l}51.78 \\
(14.44)\end{array}$ & 19.2years (13.27) & non-traumatic & Outpatient and community & IES-R \\
\hline Moodley ${ }^{61}$ & $\begin{array}{l}\text { South } \\
\text { Africa }\end{array}$ & $112(72: 40)$ & $\begin{array}{l}29.54 \\
(9.65)\end{array}$ & Up to 1 month post & Traumatic & Inpatient & IES-R; PDS \\
\hline Mona 63 & USA & $195(109: 86)$ & $\begin{array}{l}37.17 \\
(6.74)\end{array}$ & 12.2 years $(6.43)$ & Traumatic & Outpatient & PDS \\
\hline Nielsen ${ }^{10,15}$ & Denmark & $\begin{array}{l}69(-) \\
168(125: 43)\end{array}$ & $\begin{array}{l}48(16) \\
42.7(12.5)\end{array}$ & $\begin{array}{l}0.2 \text { years }(0.18) \\
14.0 \text { years }(10.1)\end{array}$ & $\begin{array}{l}\text { Traumatic and } \\
\text { non-traumatic }\end{array}$ & $\begin{array}{l}\text { Inpatient, outpatient and } \\
\text { community }\end{array}$ & HTQ \\
\hline Otis $^{3}$ & Canada & $71(56: 15)$ & $\begin{array}{l}41.06 \\
(12.27)\end{array}$ & 12.1 years $(11.52)$ & Traumatic & Outpatient & SCID \\
\hline $\begin{array}{l}\text { Radnitz }^{8,17,21,46,47} \\
\text { Danner }^{9}\end{array}$ & USA & $125(124: 1)$ & $\begin{array}{l}49.51 \\
(13.8)\end{array}$ & 18.8 years $(13.11)$ & Traumatic & Inpatient, outpatient & $\begin{array}{l}\text { IES; CAPS; } \\
\text { SCID }\end{array}$ \\
\hline Schonenberg ${ }^{24}$ & Germany & $102(86: 16)$ & $\begin{array}{l}41.31 \\
(12.6)\end{array}$ & 3.7 years $(1.79)$ & Traumatic & Outpatient & IES-R \\
\hline Ullrich 64 & USA & $87(86: 1)$ & $27.2(6.9)$ & - & Traumatic & Inpatient & PC-PTSD \\
\hline Warren ${ }^{11}$ & USA & $23(13: 10)$ & $34.5(14.4)$ & 14.3 days (18.4) & Traumatic & Inpatient & PC-PTSD \\
\hline
\end{tabular}

Measure abbreviations: CAPS Clinician Administered PTSD Scale; CPSS Child PTSD Symptom Scale; IES/IES-R Impact of Events Scale (Revised); HTQ Harvard Trauma Questionnaire; PC-PTSD Primary Care PTSD screen; PDS Posttraumatic Diagnostic Scale; PCL Posttraumatic Stress Disorder Checklist; PPTSD-R Purdue Posttraumatic Stress Disorder-Revised; SCID Structured Clinical Interview for DSM-IV/DSM-III. 


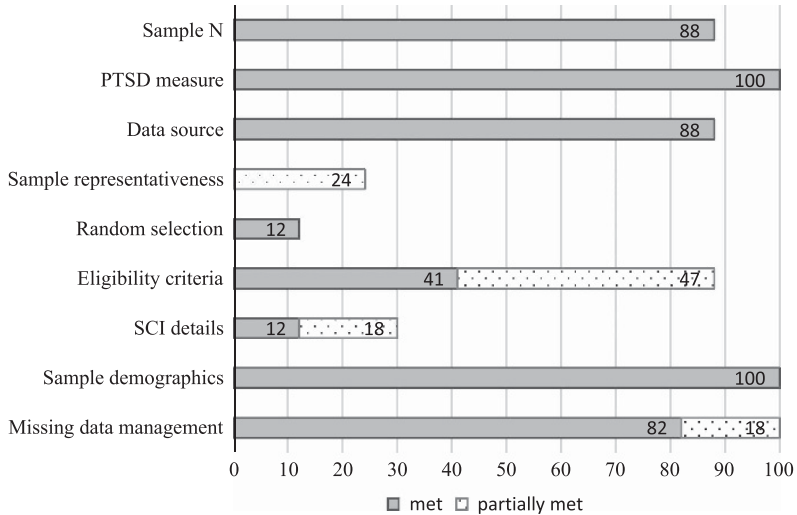

Figure 2 Risk of bias assessment.

symptomatology if it was associated with a moderate $r(r>0.10)$ that was statistically significant $(95 \% \mathrm{CIs} \neq 0, P<0.05)$ and an $N_{\mathrm{fs}}$ score that was greater than the number of studies, which contributed to the pooled effect size.

\section{RESULTS}

\section{Study characteristics}

Data from 17 studies were included in this meta-analysis. This included several studies from the United States $\left(N_{\text {studies }}=8\right)$ and Europe $\left(N_{\text {studies }}=6\right)$, with single studies from Australia, Canada and South Africa also contributing (see Table 1). Most utilised a crosssectional survey design, with Moodley and Pillay ${ }^{61}$ repeating PTSD measurements over a 4-week inpatient timeframe. Despite the broad publication range (1998-2016), the majority of PTSD measures corresponded with DSM-IV criteria: the two exceptions being the Impact of Event Scale and Clinician Administered PTSD Scale (CAPS) for DSM-III-R. All studies utilised self-report screening tools of PTSD severity. Single studies supplemented this information with full DSM criteria based on the CAPS or Structured Clinical Interview for DSM Axis I Disorders, ${ }^{2,9,18}$ although they reported findings as a continuous measure of overall PTSD symptom severity rather than as a dichotomous diagnosis.

\section{Risk of bias assessment}

As seen in Figure 2, most studies provided sufficient information relating to study design and conduct to detect potential sources of bias. Aside from two pilot studies, ${ }^{11,37}$ most were also sufficiently powered $\left(N=26\right.$, power at $\left.0.80, \alpha=0.05, r=0.50 ;{ }^{62}\right)$. The requirement that included studies utilise robust PTSD tools and primary data sources also minimised measurement error. However, the majority relied on a single recruitment source (for example, single rehabilitation unit), as opposed to broad recruitment strategies (for example, electronic advertising, postal mail and so on), which may limit the degree to which the findings can be generalised to the broader SCI population. Chung et al. ${ }^{13,39,40}$ and Mona et al. ${ }^{63}$ incorporated some form of random selection for their recruitment (for example, computergenerated sampling). Inclusion and exclusion criteria were routinely reported, with potential participants with preexisting medical conditions (for example, stroke, psychosis) or associated head trauma excluded. Critical sample character parameters (that is, age, gender) were provided by all studies, although few referred to international standards (ASIA Impairment Scale, Frankel Scale) for the neurological classification of SCI. Finally, missing data were at least partially explained by all studies (that is, by providing $N_{\text {participants }}$ per measure or $N$ incomplete data), thereby minimising the risk of potential attrition bias.

\section{Participant characteristics}

The total pooled sample comprised 2980 individuals with an acquired SCI (see Table 2), most assessed as adults. Boyer's family study ${ }^{37,38}$ exclusively focussed on paediatric SCI. The male-to-female ratio of 3:1 is consistent with reported SCI prevalence data, ${ }^{7}$ with individuals primarily injured in motor vehicle crashes. Eleven studies exclusively recruited those with a traumatic injury onset with three independent studies (Radnitz et al. ${ }^{8,9,17,21,46,47}$; Martz et al. ${ }^{14,43}$; Ullrich et al. ${ }^{64}$ ) examining PTSD associations in veteran groups. Two studies screened for PTSD in the acute phase of a SCI trauma. ${ }^{11,61}$

\section{Effect-size estimates}

Individual and pooled $r$ for 18 PTSD risk factors are listed in Table 3 and rank ordered by size. The weighted effect estimates for five factors, all post-injury variables, can be considered clinically important in accordance with the following criteria adopted for this review (that is, $r>0.10 ; N_{\mathrm{fs}}>N$; CIs $\neq 0, P<0.05$ ): depression comorbidity, posttraumatic cognitions, psychological distress, anxiety, and pain level.

\section{Pre-trauma factors}

Single studies identified a strong correlation between prior history variables and PTSD symptomatology. The experience of psychiatric illness (defined as a single-item question on history of clinically diagnosed affective, anxiety, substance abuse or psychotic disorders) was a positive risk factor for patients reporting distress in the acute trauma setting. ${ }^{11}$ In the longer term, those who had experienced an adverse life event ${ }^{2}$ prior to injury were at greater risk of developing PTSD (Table 3). However, pooled estimates for these variables did not reach significance. A fairly consistent pattern of gendered differences in symptom severity was reported, with women reporting more severe symptomatology, both in the acute and chronic stages of SCI. ${ }^{3,12,41}$ Nonsignificant associations were found for the remaining sociodemographic variables.

\section{Peri-trauma factors}

Pooled data revealed a small-to-medium association between SCI-related factors and PTSD aetiology, although the direction of effects varied (Table 3). Adults with a complete lesion reported significantly more symptoms of hyperarousal, flashbacks and avoidance. ${ }^{2,12,41}$ Although Nielsen et al. ${ }^{10}$ reported a similar finding among their sample of inpatients, they identified injury completeness as a key variable, which mitigated the development of PTSD in a larger-scale study. ${ }^{15}$ Other sociomedical indices-alcohol or substance use at the time of injury, age at injury, loss of consciousness and injury type-did not correlate significantly with PTSD, although these findings were based on limited data. ${ }^{2,11,15,17}$ Notably, SCI type was associated with a broad range of effect-size estimates: adults with paraplegia reported heightened distress $(r=-0.37$ (95\% CI, -0.51 to -0.21), $P<0.01),{ }^{8}$ whereas a higher percentage of individuals with tetraplegia reported a moderate level of acute stress symptoms. ${ }^{61}$ In addition, avoidance symptoms were common among children and adolescents with tetraplegic injuries $(r=0.51$, (95\% CI, 0.10-0.77), $P=0.02),{ }^{37}$ although this finding may be confounded by behavioural changes associated with reduced physical capabilities in the younger cohort. $^{37,38}$ 
Table 2 Sample characteristics $\left(N_{\text {participants }}=2980\right)$

\begin{tabular}{|c|c|c|c|c|}
\hline Variable & $\mathrm{N}_{\text {studies }}$ & $\mathrm{N}_{\text {participants }}(\%)$ & Mean (s.d.) & Range \\
\hline Sample size & 17 & $2980(100)$ & $175.3(221.1)$ & $21-927$ \\
\hline $\begin{array}{l}\text { Age at study recruitment } \\
\text { (years) }\end{array}$ & 15 & $2032(68)$ & $41.0(7.7)$ & $27-52$ \\
\hline $\begin{array}{l}\text { Age at the time of injury } \\
\text { (years) }\end{array}$ & 9 & 2037 (68) & $29.6(8.4)$ & $12-39$ \\
\hline Time since injury (years) & 11 & $2646(89)$ & $12.8(6.4)$ & $0.02-22.5$ \\
\hline \multicolumn{5}{|l|}{ Education } \\
\hline Years & 6 & $937(31)$ & $11.5(2.7)$ & $6-13.6$ \\
\hline \multicolumn{5}{|l|}{ Gender } \\
\hline Male & 17 & $2270(76)$ & & \\
\hline Female & 17 & $709(24)$ & & \\
\hline \multicolumn{5}{|l|}{ Marital status } \\
\hline Single/widowed & 11 & $810(27)$ & & \\
\hline Married/partnered & 11 & $765(26)$ & & \\
\hline \multicolumn{5}{|l|}{ Employment status } \\
\hline Employed & 3 & $144(5)$ & & \\
\hline Unemployed & 3 & $164(6)$ & & \\
\hline \multicolumn{5}{|l|}{ Type/level of SCl } \\
\hline Tetraplegia & 14 & $899(30)$ & & \\
\hline Paraplegia & 14 & $727(24)$ & & \\
\hline \multicolumn{5}{|l|}{ Completeness of injury } \\
\hline Complete & 12 & $878(29)$ & & \\
\hline Incomplete & 12 & $841(28)$ & & \\
\hline \multicolumn{5}{|l|}{ Nature of injury } \\
\hline Traumatic & 13 & $2427(81)$ & & \\
\hline Nontraumatic & 2 & $62(2)$ & & \\
\hline \multicolumn{5}{|l|}{ Injury cause } \\
\hline Motor vehicle accident & 15 & 1167 (39) & & \\
\hline Fall & 13 & $367(12)$ & & \\
\hline Sports related & 10 & $249(8)$ & & \\
\hline Violent & 10 & $279(9)$ & & \\
\hline Other & 14 & $294(10)$ & & \\
\hline
\end{tabular}

Abbreviation: $\mathrm{SCl}$, spinal cord injury.

$N_{\text {studies }}=$ number of studies providing data; $N_{\text {participants }}=$ number of participants providing this data.

\section{Post-trauma factors}

Medium-to-large effect-size estimates were noted by several studies that evaluated the cross-sectional association between PTSD and SCI sequelae. Indices of psychological adjustment were strongly related: those who experienced negative emotions and cognitions related to their SCI (that is, a reflection of lower levels of acceptance/adjustment) reported severe PTSD symptoms (Table 3). Although depression, as a construct, was characterised by between-study heterogeneity, potentially reflecting its multidimensional nature (that is, as a feature of general psychological distress ${ }^{6}$ or a case-finding instrument ${ }^{65}$ ), the pooled weighted effect estimate $(r>0.50)$ was highly significant given the large $N$ involved. Those reporting daily or severe neuropathic pain also experienced intense psychological distress. ${ }^{2,43}$ There was also a trend for those in the acute stages of SCI rehabilitation to report subtle PTSD symptoms, although this finding was characterised by publication bias.

Seven independent studies examined the relative contribution of perceived and actual support (Table 3). Although medium-to-large effect estimates $(r>0.30$; Table 3$)$ for perceived satisfaction were noted-those who expressed greater satisfaction with the emotional and instrumental support provided by close family and friends endorsed fewer PTSD symptoms-this finding was primarily based on single studies. Similarly, Chung et al. ${ }^{13}$ identified a strong relationship between the ability to carry out social functions and hyperarousal $(r=0.49$ (95\% CI, 0.27-0.66) $P<0.01)$, re-experiencing $(r=0.44 \quad(95 \%$ CI, $0.21-0.62) \quad P<0.01)$ and avoidance $(r=0.41$ (95\% CI, 0.18-0.60) $P<0.01)$ symptoms. In comparison, well-being was not significantly associated with the more support that was received $d^{2,3,9,12}$ nor attempts to actively seek support. ${ }^{45}$ In combination, these findings highlight the empirical distinctness of perceived social support from social network size.

\section{Moderator analyses}

Analysis by measure and sample type was conducted for the following three risk factors: SCI severity, age at injury and SCI level. This yielded significant results for one risk factor only: veterans with paraplegia were at risk for PTSD $\left(Q_{\mathrm{B}}(1)=18.25, P<0.01\right.$; veterans $r=-0.37$ (95\% CI, -0.57 to -0.21 ) $P<0.01$; civilians $r=0.10$ (95\% CI, -0.04 to 0.24$) P=0.15)$. However, given that this subgroup analysis was based on a limited dataset ${ }^{9,17,43}$, any between-studies variance identified is likely to have poor precision. ${ }^{57}$ Group equivalence could not be compared for depression, as a risk factor, as measurement and sample characteristics did not vary between studies.

\section{DISCUSSION}

This meta-analytic review examined potential risk factors associated with PTSD development following an acquired SCI. The combined findings help refine current understanding of the aetiology of PTSD following SCI, with postinjury adjustment issues likely to increase an individual's vulnerability to distress. The summary evidence also helps to profile types of SCI studies examining PTSD and aspects needing further enquiry.

In contrast to previous reviews of trauma-exposed adults, pooled effect estimates suggest that preexisting socioenvironmental factors-namely, prior trauma exposure, ${ }^{28,66}$ higher education ${ }^{31}$ and spousal support ${ }^{67}$ - did not significantly influence risk of PTSD development, at least for the SCI sample examined in this review. Notably, the number of studies involved in these analyses was limited in quantity, underlining the need for further research before drawing any firm conclusions on pretrauma risk factors. This includes the potential contribution of age: the younger the age of trauma exposure, the more potent its effect on future PTSD psychopathology. ${ }^{68}$ The impact of trauma severity (that is, trauma uniqueness, stressfulness) and type on PTSD development also requires further examination. ${ }^{17,31,33}$ Within the civilian SCI population, however, these relationships have not been identified. To help inform a diathesisstress formulation of PTSD, future SCI studies might also consider examining concurrent influences of biological diatheses. Emerging evidence suggest that cognitive impairment, in particular, can impede an individual's ability to process and manage a traumatic experience such as SCI. ${ }^{62,69}$ Notably, loss or impairment of consciousness at the time of injury, which may effect subsequent cognitive and psychological functioning, ${ }^{70}$ was not consistently associated with PTSD symptom severity in this review. 
Table 3 Risk factors for post-traumatic stress listed by individual study

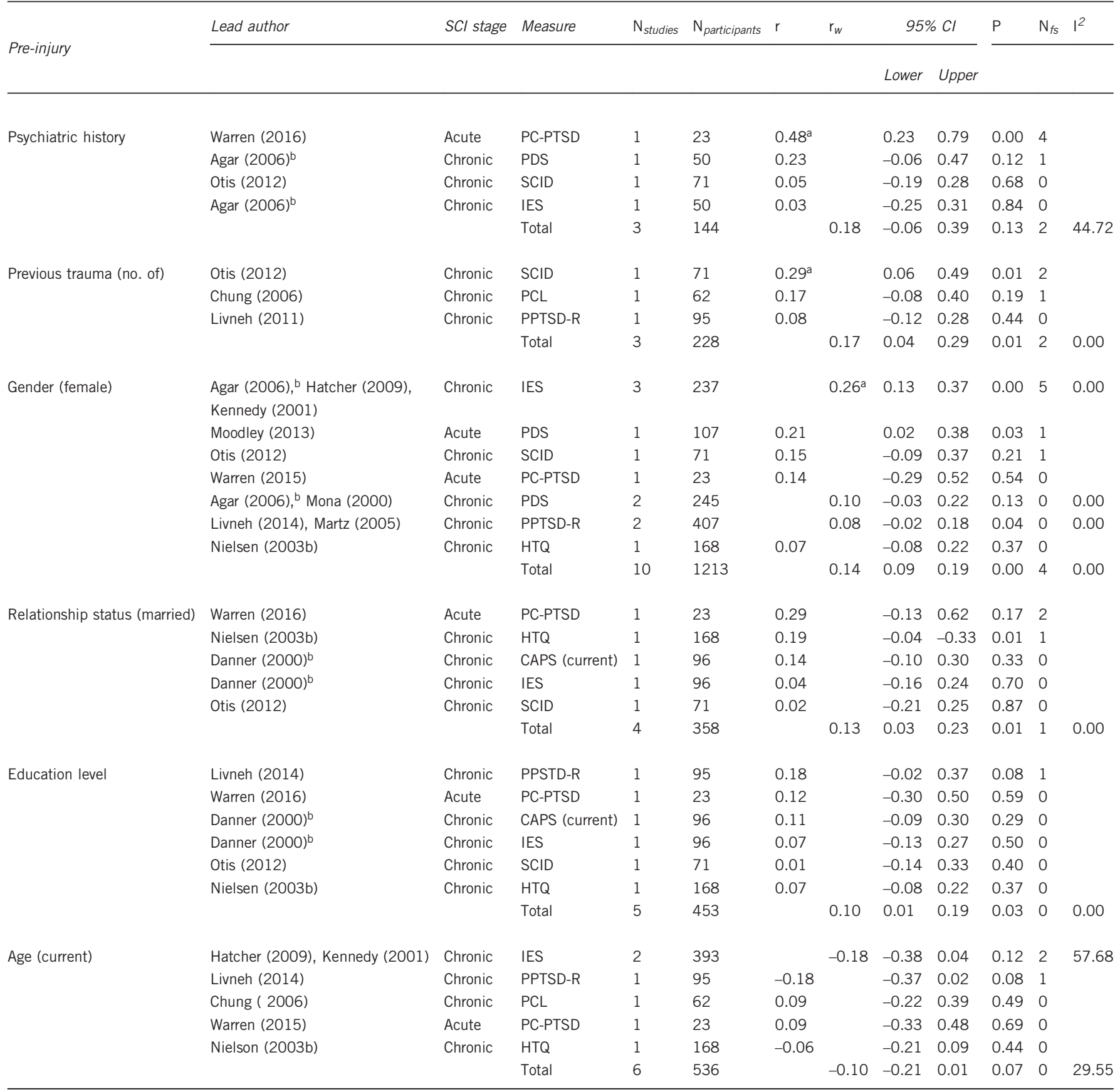

Peri-injury

\begin{tabular}{|c|c|c|c|c|c|c|c|c|c|c|c|c|}
\hline $\mathrm{SCl}$ severity (completeness) & Otis (2012) & Chronic & SCID & 1 & 71 & $0.29^{a}$ & & 0.06 & 0.49 & 0.01 & 2 & \\
\hline & Agar (2006), b Kennedy (2001) & Chronic & IES & 1 & 135 & $0.28^{\mathrm{a}}$ & & 0.00 & 0.53 & 0.05 & 2 & \\
\hline & Nielsen (2003b) & Chronic & HTQ & 1 & 168 & -0.17 & & -0.31 & -0.02 & 0.03 & 1 & \\
\hline & & & Total & 5 & 569 & & 0.12 & -0.06 & 0.29 & 0.21 & 1 & 77.39 \\
\hline \multirow[t]{2}{*}{ Alcohol/substance use } & Warren (2016) & Acute & PC-PTSD & 1 & 24 & 0.08 & & -0.33 & 0.47 & 0.71 & 0 & \\
\hline & & & Total & 2 & 149 & & 0.05 & -0.21 & 0.10 & 0.52 & 1 & 0.00 \\
\hline
\end{tabular}


Table 3 (Continued)

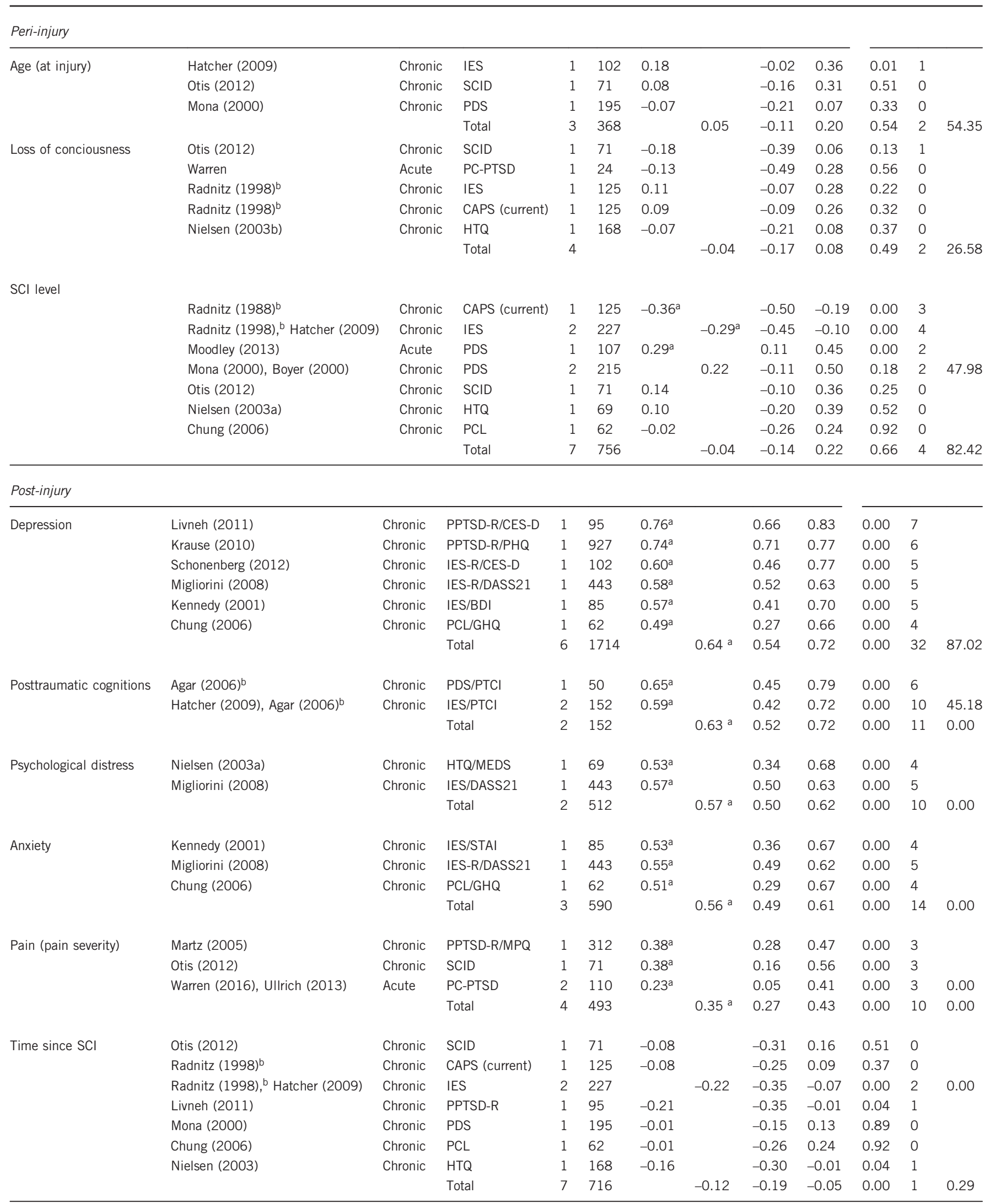


Table 3 (Continued)

\begin{tabular}{|c|c|c|c|c|c|c|c|c|c|c|c|c|c|}
\hline \multirow[t]{2}{*}{ Risk factor } & & \multirow[t]{2}{*}{ Lead author (date) } & \multirow[t]{2}{*}{ SCl stage } & \multirow[t]{2}{*}{ Measure(s) } & \multirow[t]{2}{*}{$\mathrm{N}_{\text {studies }}$} & \multirow[t]{2}{*}{$\mathrm{N}_{\text {participants }}$} & \multirow[t]{2}{*}{ r } & \multirow[t]{2}{*}{$r_{w}$} & \multicolumn{2}{|c|}{$95 \% \mathrm{Cl}$} & \multirow[t]{2}{*}{$P$} & \multirow[t]{2}{*}{$\mathrm{N}_{f s}$} & \multirow[t]{2}{*}{$\mathrm{I}^{2}$} \\
\hline & & & & & & & & & Lower & Upper & & & \\
\hline & & Agar (2006) & Chronic & PDS/PSS & 1 & 50 & $-0.39^{a}$ & & -0.60 & -0.12 & 0.01 & 3 & \\
\hline & & Agar (2006) & Chronic & IES/PSS & 1 & 50 & $-0.36^{a}$ & & -0.58 & -0.09 & 0.01 & 3 & \\
\hline & Social dysfunction & Chung (2006) & Chronic & $\mathrm{PCL} / \mathrm{GHQ}$ & 1 & 62 & $0.45^{\mathrm{a}}$ & & 0.23 & 0.63 & 0.00 & 4 & \\
\hline & & Danner (2000) & & & & & & & & & & & \multirow{5}{*}{0.00} \\
\hline & & Danner (2000) & Chronic & CAPS/PSS & 1 & 124 & -0.15 & & & & & 1 & \\
\hline & & Agar (2006) & Chronic & $\mathrm{PDS} / \mathrm{SSQ}$ & 1 & 50 & -0.04 & & -0.31 & 0.25 & 0.81 & 0 & \\
\hline & & Agar (2006) & Chronic & $\mathrm{IES} / \mathrm{SSQ}$ & 1 & 50 & -0.02 & & -0.26 & 0.29 & 0.91 & 0 & \\
\hline & Seeking support & Livneh (2011) & Chronic & PPTSD-R/COPE & 1 & 95 & -0.12 & & -0.31 & 0.08 & 0.25 & 0 & \\
\hline
\end{tabular}

Note: $N_{\text {studies }}=$ number of studies providing data; $N_{\text {participants }}=$ number of participants providing this data; $r_{\mathrm{w}}=$ weighted mean correlation; $95 \% \mathrm{Cl}=\mathrm{confidence}$ interval with lower $(\mathrm{L})$ and upper $(\mathrm{U})$ Note: $N_{\text {studies }}=$ number of studies providing data;

Measure abbreviations: BDI Beck Depression Inventory; CES-D; CSS Crisis Support Scale; COPE, COPE Inventory; DASS-21 DASS21, Depression Anxiety Stress Scales-21 item; GHQ General Health Questionnaire; MPQ McGill Pain Questionnaire; MEDS, Medical-Based Emotional Distress; PHQ Patient Health Questionnaire; PNSBS Perceived Negative Spouse Behaviors Scale; PSS Perceived Social Support Scale; PTCI Post-traumatic cognitions inventory; SSQ Short Form Social Support Questionnaire; STAI StateTrait Anxiety Inventory.

${ }^{a}$ significant effect size: $r>0.10, \mathrm{Cl} \neq 0, N f s>N_{\text {studies }}, P<0.05, N f s>N$ studies, ${ }^{b}$ studies providing more than one effect per factor were averaged for overall effect size calculation.

Although a sizeable group of SCI studies have examined the contribution of post-SCI factors in increasing or decreasing the risk of developing PTSD, there remains little available data relating to the core trauma that may trigger PTSD. Recent studies suggest that factors related to the acute hospital experience (for example, exposure to an intensive care unit, sedation level, intubation and reintubation and length of stay) contribute to a unique constellation of PTSD symptoms, including sleep problems. ${ }^{71-73}$ Notably, the finding that psychological risk factors (that is, negative affect, general distress, depression and anxiety) were critical to PTSD was consistent across the examined studies. Depression and anxiety are known to mediate stress reactions in the trauma population. ${ }^{74,75}$ However, it remains unclear whether these mental health problems were preexisting or co-occurring in response to SCI as contributing studies did not routinely examine pre-morbid psychiatric illness. Symptom overlap between PTSD and depression also warrant consideration, with arousal (for example, sleep problems) and avoidance (for example, loss of interest in usual activities) being characteristic of both. ${ }^{76}$ Moreover, cognitive responses to trauma such as dissociation reactions, self-blame and negative cognitions of the world, although well established in the general PTSD literature, ${ }^{77}$ require further investigation with the SCI cohort.

That PTSD severity is enhanced by pain is consistent with current theory that pain sensations serve as a constant reminder of trauma, triggering PTSD re-experiencing symptoms. ${ }^{7}$ It is thought that pain contributes to the development and maintenance of PTSD. ${ }^{78}$ The importance of familial relationships in maintaining mental health was also highlighted in this review. ${ }^{15}$ Balanced and nurturing personal relationships are particularly important for young injured persons as they help to foster encouragement, expectations and independence, helping to buffer distress associated with SCI as a traumatic event. ${ }^{37,38}$ In comparison, family instability (that is, history of mental health) may increase the risk of PTSD. ${ }^{37,38}$ Notably, SCI studies have not routinely considered how differences in the structure and function of perceived supports (for example, informational, tangible or emotional supports) may have different mental health implications.

\section{Clinical implications}

The combined findings, which suggest that PTSD following SCI involves a complex mix of life experiences, inherited mental health risks and coping capabilities, underscore the importance of efficacious screening tools targeted to this patient cohort. One promising tool is the 19-item Spinal Cord Injury-Quality of Life Psychological Trauma item bank. ${ }^{79}$ Another emerging measure is the DSM-5 Cross-Cutting Symptoms Measure, designed to assess the presence and severity of co-occurring psychological disorders or symptoms, ${ }^{80}$ although the feasibility of this DSM-5 measure in SCI practice and research is still to be determined.

Multi-domain interventions in the acute stages of SCI rehabilitation can prevent subsequent psychopathology for individuals identified as having high-stress diathesis. ${ }^{81}$ This might include cognitivebehavioural programmes, ${ }^{82,83}$ which have shown promise as an approach for managing adjustment problems, such as depression and anxiety. Psychoeducation is another critical therapy component for individuals at risk of developing PTSD. Even basic information about trauma-related symptoms can help to reduce misconceptions of mental illness and influence psychological recovery in a positive way. ${ }^{84}$ This informational support can be supplemented with social support networking skills. This includes capitalising on the quality of support within existing social networks in addition to fostering new connections for those who are socially isolated. ${ }^{2,65,85}$ Rehabilitation strategies that reinforce injury health behaviours, including coping selfstatements and proactive strategies for managing chronic pain, can also help protect against trauma-induced psychopathology. ${ }^{2}$

\section{Study limitations}

The present findings need to be considered in the context of a number of important limitations. First, the search criteria may have failed to capture all relevant studies. In an attempt to minimise this limitation, multiple search strategies (for example, electronic database searches, manual search of reference lists of reviews, contacting experts in the topic) were adopted. Further, $N_{\mathrm{fs}}$ statistics were calculated. It is acknowledged, however, that this statistic does not fully alleviate the problem of publication bias. ${ }^{59}$ 
Second, the tendency for SCI studies to use overlapping data (for example, the same original patient sample, or secondary analyses of data that cover the same outcomes or overlapping subsets of them) presents statistical difficulties for meta-analysis methods. ${ }^{36}$ Importantly, through a careful search of similar studies, data independence was ensured. Although this resulted in a reduced sample, none of the observations overlapped, and no single study provided a disproportionate amount of data to the calculation of a pooled effect size $r$. To ensure transparency of data, it is nonetheless important for SCI substudies to cite any primary publication.

Third, the majority of the data were self-reports, which are prone to recall, confounding and sample selection biases. ${ }^{86}$ Notably, individual effect sizes did not markedly differ across individual PTSD measures. This included similar effect estimates produced with the original and revised Impact of Event Scale, despite the former's two symptom (that is, intrusion, avoidance) cluster conceptualisation of PTSD, which no longer matches DSM diagnostic criteria. ${ }^{76}$ Nonetheless, the current findings need to be interpreted with caution given the reliance on earlier DSM versions. The current DSM-5 incorporates a four-symptom model that considers alterations in mood and cognitions (for example, negative beliefs, negative emotional state) and may enable more accurate diagnosis of PTSD in the SCI cohort. ${ }^{87}$ Indeed, cognitive and affective symptoms are just as critical to the experience of PTSD, following SCI, as the experience of physiological (arousal) symptoms. ${ }^{12}$

Fourth, as the majority of studies were conducted in Western countries, the extrapolation of findings may be limited. Although the diagnosis of PTSD is cross-culturally valid, it is acknowledged in the DSM-5 that expression of PTSD and symptom comorbidities may differ across cultures. ${ }^{88}$ Fifth, the cross-sectional and retrospective nature means that the examined variables were, for the most part, assessed at a single time point and may be confounded by prior symptoms and functioning. ${ }^{89}$ There is also evidence that traumatic symptoms are dynamic and can vary in intensity over time. ${ }^{2,90}$ Indeed, recent data suggest that demographic (for example, less years of education, not partnered) and injury variables (for example, lesion completeness) correctly predict the likelihood of developing a mental disorder up to 6 months after discharge. ${ }^{2}$ In order to determine the temporality between PTSD risk factors for those with SCI, longitudinal studies driven by articulated biopsychosocial models of causation-such as the diathesis-stress model-are needed. Attempts to minimise potential sources of bias in sampling, selection, measurement and data analysis will also help to ensure that the results are generalisable and translational. ${ }^{90}$

\section{CONCLUSION}

This meta-analytic review exposed significant relationships between several postinjury risk factors and PTSD symptoms following SCI. The suggestion is that attention needs to be given to the role of personal variables in the identification and management of posttraumatic stress symptoms in this cohort. Issues of further study were also identified, including the need to examine sociocontextual and biological factors that may contribute to high-stress diathesis.

\section{DATA ARCHIVING}

There were no data to deposit.

\section{CONFLICT OF INTEREST}

The authors declare no conflict of interest.

\section{ACKNOWLEDGEMENTS}

We thank M Bell, Research Librarian at the University of Adelaide, for helping develop the database logic grids for searching. We also thank the authors of included studies who kindly provided additional statistical information on request.

References marked with an asterisk $\left(^{*}\right)$ indicate studies included in the meta-analysis.

1 Kennedy P, Duff J. Post traumatic stress disorder and spinal cord injuries. Spinal Cord 2001; 39: 1-10.

2 Craig A, Nicholson Perry K, Guest R, Tran Y, Dezarnaulds A, Hales A et al. Prospective study of the occurrence of psychological disorders and comorbidities after spinal cord injury. Arch Phys Med Rehabil 2015; 96: 1426-1434.

3 * Otis C, Marchand A, Courtois F. Risk factors for posttraumatic stress disorder in persons with spinal cord injury. Top Spinal Cord Inj Rehabil 2012; 18: 253-263.

$4{ }^{*}$ Hatcher MB, Whitaker C, Karl A. What predicts post-traumatic stress following spinal cord injury. Br J Health Psychol 2009; 14: 541-561.

5 Livneh H, Martz E. The impact of perceptions of health control and coping modes on negative affect among individuals with spinal cord injuries. J Clin Psychol Med Settings 2011; 18: 243-256.

6 *Migliorini C, Tonge B, Taleporos G. Spinal cord injury and mental health. Aust N Z J Psychiatry 2008; 42: 309-314.

7 Singh A, Tetreault L, Kalsi-Ryan S, Nouri A, Fehlings MG. Global prevalence and incidence of traumatic spinal cord injury. Clin Epidemiol 2014; 6: 309-331.

8 *Radnitz CL, Schlein IS, Hsu L. The effect of prior trauma exposure on the development of PTSD following spinal cord injury. J Anxiety Disord 2000; 14: 313-324.

9 *Danner G, Radnitz CL. Protective factors and posttraumatic stress disorder in veterans with spinal cord injury. Int J Rehabil Health 2000; 5: 195-203.

10 *Nielsen MS. Post-traumatic stress disorder and emotional distress in persons with spinal cord lesion. Spinal Cord 2003a; 41: 296-302.

11 *Warren AM, Reynolds M, Driver S, Bennett M, Sikka S. Posttraumatic stress symptoms among spinal cord injury patients in trauma: a brief report. Top Spinal Cord Inj Rehabil 2016; 22: 203-208.

12 *Agar E, Kennedy P, King NS. The role of negative cognitive appraisals in PTSD symptoms following spinal cord injuries. Behav Cogn Psychother 2006; 34: 437-452.

13 *Chung MC, Preveza E, Papandreou K, Prevezas N. Locus of control among spinal cord injury patients with different levels of posttraumatic stress disorder. Psychiatry Res 2007; 152: 253-260.

14 *Martz E. Death anxiety as a predictor of posttraumatic stress levels among individuals with spinal cord injuries. Death Stud 2004; 28: 1-17.

15 *Nielsen MS. Prevalence of posttraumatic stress disorder in persons with spinal cord injuries: the mediating effect of social support. Rehabil Psychol 2003b; 48: 289-295.

16 Khazaeipour Z, Taheri-Otaghsara S-M, Naghdi M. Depression following spinal cord injury: its relationship to demographic and socioeconomic indicators. Top Spinal Cord Inj Rehabil 2015; 21: 149-155.

17 *Radnitz CL, Hsu L, Willard J, Perez-Strumolo L, Festa J, Lillian LB et al. Posttraumatic stress disorder in veterans with spinal cord injury: trauma-related riskfactors. J Trauma Stress 1998; 11: 505-520.

18 Marin I. Societal Attitudes and Myths about Disabilities: Improving the Socia Consciousness. In: Noreen M. Glover-Graf RhD CRC, Michael Millington PhD CRC (eds), Psychosocial Aspects of Disability: Insider Perspectives and Strategies for Counselors. Springer Publishing Company, New York, NY, 2011, pp 33-60.

19 Ullrich PM, Smith BM, Blow FC, Valenstein M, Weaver FM. Depression, healthcare utilization and comorbid psychiatric disorders after spinal cord injury. J Spinal Cord Med 2014; 37: 40-45.

20 Breslau N, Chilcoat HD, Kessler RC, Davis GC. Previous exposure to trauma and PTSD effects of subsequent trauma: results from the Detroit Area Survey of Trauma. Am J Psychiatry 1999; 156: 902-907.

21 *Radnitz CL, Hsu L, Tirch DD, Willard J, Lillian LB, Walczak S et al. A comparison of posttraumatic stress disorder in veterans with and without spinal cord injury. J Abnorm Psychol 1998; 107: 676-680.

22 Jensen MP, Moore MR, Bockow TB, Ehde DM, Engel JM. Psychosocial factors and adjustment to chronic pain in persons with physical disabilities: a systematic review. Arch Phys Med Rehabil 2011; 92: 146-160.

23 Tran J, Dorstyn D, Burke A. Psychosocial aspects of spinal cord injury pain: a metaanalysis. Spinal Cord 2016; 54: 640-648.

24 *Schönenberg M, Reimitz M, Jusyte A, Maier D, Badke A, Hautzinger M. Depression, posttraumatic stress, and risk factors following spinal cord injury. Int J Behav Med 2014; 21: 169-176.

25 Haidich AB. Meta-analysis in medical research. Hippokratia 2010; 14(Suppl 1): 29-37.

26 Middleton J, Craig A. In: Craig A, Tran Y (eds). Psychological Dynamics Associated with Spinal Cord Injury Rehabilitation: New Directions and Best Evidence. Nova Science Publishers: New York, USA, 2008, pp 3-53.

27 Craig A, Tran Y, Middleton J. Psychological morbidity and spinal cord injury: a systematic review. Spinal Cord 2009; 47: 108-114.

28 Ozer EJ, Best SR, Lipsey TL, Weiss DS. Predictors of posttraumatic stress disorder and symptoms in adults: a meta-analysis. Psychol Bull 2003; 129: 52-73. 
29 Post MWM, van Leeuwen CMC. Psychosocial issues in spinal cord injury: a review. Spinal Cord 2012; 50: 382-389.

30 North N. The psychological effects of spinal cord injury: a review. Spinal Cord 1999; 37: 671-679.

31 Brewin CR, Andrews B, Valentine JD. Meta-analysis of risk factors for posttraumatic stress disorder in trauma-exposed adults. J Consult Clin Psychol 2000; 68: 748-766.

32 Trickey D, Siddaway AP, Meiser-Stedman R, Serpell L, Field AP. A meta-analysis of risk factors for post-traumatic stress disorder in children and adolescents. Clin Psychol Rev 2012; 32: 122-138.

33 Xue C, Ge Y, Tang B, Liu Y, Kang P, Wang M et al. A meta-analysis of risk factors for combat-related PTSD among military personnel and veterans. PLOS ONE 2015; 10: e0120270.

34 Jüni P, Holenstein F, Sterne J, Bartlett C, Egger M. Direction and impact of language bias in meta-analyses of controlled trials: empirical study. Int J Epidemiol 2002; 31 115-123.

35 Valentine JC, Pigott TD, Rothstein HR. How many studies do you need? A primer on statistical power for meta-analysis. J Educ Behav Stat 2010; 35: 215-247.

36 Lipsey MW, Wilson DB. Practical Meta-Analysis. SAGE Publications, Inc.: Thousand Oaks, California, USA, 2001

37 * Boyer BA, Tollen LG, Kafkalas CM. A pilot study of posttraumatic stress disorder in children and adolescents with spinal cord injury. SCI Psychosocial Process 1998; 11: 75-81.

38 *Boyer BA, Knolls ML, Kafkalas CM, Tollen LG, Swartz M. Prevalence and relationships of posttraumatic stress in families experiencing pediatric spinal cord injury. Rehabil Psychol 2000; 45: 339-355.

$39{ }^{*}$ Chung MC, Preveza E, Papandreou K, Prevezas N. The relationship between posttraumatic stress disorder following spinal cord injury and locus of control. J Affect Disord 2006; 93: 229-232.

40 *Chung MC, Preveza E, Papandreou K, Prevezas N. Spinal cord injury, posttraumatic stress, and locus of control among the elderly: a comparison with young and middle - aged patients. Psychiatry 2006; 69: 69-80.

41 *Kennedy P, Evans MJ. Evaluation of post traumatic distress in the first 6 months following SCl. Spinal Cord 2001; 39: 381-386.

42 *Lude P, Kennedy P, Evans M, Lude Y, Beedie A. Post traumatic distress symptoms following spinal cord injury: a comparative review of European samples. Spinal Cord 2005; 43: 102-108.

43 *Martz E. Associations and predictors of posttraumatic stress levels according to person-related, disability-related, and trauma-related variables among individuals with spinal cord injuries. Rehabil Psychol 2005; 50: 149-157.

44 *Martz E, Livneh H, Priebe M, Wuermser LA, Ottomanelli L. Predictors of psychosocial adaptation among people with spinal cord injury or disorder. Arch Phys Med Rehabil 2005; 86: 1182-1192.

45 *Livneh H, Martz E. Coping strategies and resources as predictors of psychosocial adaptation among people with spinal cord injury. Rehabil Psychol 2014; 59: 329-339.

46 *Binks T, Radnitz CL, Moran Al, Vinciguerra V. Relationship between level of spinal cord injury and posttraumatic stress disorder symptoms. Ann N Y Acad Sci 1997; 821: 430-432.

$47{ }^{*}$ Goldman RL, Radnitz C, McGrath RE. Posttraumatic stress disorder and major depression in veterans with spinal cord injury. Rehabil Psychol 2008; 53: 162-170.

48 Sanderson S, Tatt ID, Higgins JP. Tools for assessing quality and susceptibility to bias in observational studies in epidemiology: a systematic review and annotated bibliography. Int J Epidemiol 2007; 36: 666-676.

49 Shamliyan TA, Kane RL, Ansari MT, Raman G, Berkman ND, Grant M et al. Development quality criteria to evaluate nontherapeutic studies of incidence, prevalence, or risk factors of chronic diseases: pilot study of new checklists. J Clin Epidemiol 2011; 64: 637-657.

50 Viswanathan M, Ansari MT, Berkman ND, Chang S, Hartling L, McPheeters M et al. Assessing the Risk of Bias of Individual Studies in Systematic Reviews of Health Care Interventions. Methods Guide for Effectiveness and Comparative Effectiveness Reviews. Agency for Healthcare Research and Quality (US): Rockville (MD), USA, 2008.

51 Dorstyn D, Black R, Mpofu E, Kneebone I. Utilizing the ICF to understand depressive symptomology in multiple sclerosis: an exploratory systematic review. Rehabil Psychol 2017, http://dx.doi.org/10.1037/rep0000125

52 Pannucci CJ, Wilkins EG. Identifying and avoiding bias in research. Plast Reconstr Surg 2010; 126: 619-625.

53 Moher D, Liberati A, Tetzlaff J, Altman DG. Preferred reporting items for systematic reviews and meta-analyses: the PRISMA statement. Int J Surg 2010; 8: 336-341.

54 Ellis PD. The Essential Guide to Effect Sizes: Statistical Power, Meta-analysis, and the Interpretation of Research Results. Cambridge University Press: Cambridge, UK, 2010.

55 Rosenthal R, DiMatteo MR. Meta-analysis: recent developments in quantitative methods for literature reviews. Annu Rev Psychol 2001; 52: 59-82.

56 Cohen J. A power primer. Psychol Bull 1992; 112: 155-159.

57 Borenstein M, Hedges LV, Higgins JPT, Rothstein HR. Introduction to Meta-analysis. John Wiley \& Sons: West Sussex, UK; Hoboken, NJ, USA, 2009.

58 Stratford PW. The added value of confidence intervals. Phys Ther 2010; 90: 333-335

59 Orwin RG. A fail-safe N for effect size in meta-analysis. J Educ Behav Stat 1983; 8: 157-159.

60 Higgins JP, Thompson SG, Deeks JJ, Altman DG. Measuring inconsistency in metaanalyses. BMJ 2003; 327: 557-560.
61 * Moodley N, Pillay BJ. Post-traumatic stress disorder in patients with spinal-cord injuries. S Afr J Psychol 2013; 43: 182-197.

62 Faul F, Erdfelder E, Lang A-G, Buchner A. GPower 3: a flexible statistical power analysis program for the social, behavioural, and biomedical sciences. Behav Res Methods 2007; 39: 175-191.

63 *Mona L, Cameron R, Lesondak L, Norris F. Posttraumatic stress disorder symptomatology in men and women with spinal cord injury. Top Spinal Cord Inj Rehabil 2000; 6: 76-86.

64 *Ullrich PM, Smith BM, Poggenesee L, Evans CT, Stroupe KT, Weaver FM et al. Pain and post-traumatic stress disorder symptoms during inpatient rehabilitation among operation enduring freedon/operation Iraqi freedom veterans with spinal cord injury. Arch Phys Med Rehabil 2013; 94: 80-85.

65 * Krause JS, Saunders LL, Newman S. Posttraumatic stress disorder and spinal cord injury. Arch Phys Med Rehabil 2010; 91: 1182-1187.

66 Heron-Delaney M, Kenardy J, Charlton E, Matsuoka Y. A systematic review of predictors of posttraumatic stress disorder (PTSD) for adult road traffic crash survivors. Injury 2013; 44: 1413-1422.

67 Kessler RC, Sonnega A, Bromet E, Hughes M, Nelson CB. Posttraumatic stress disorder in the National Comorbidity Survey. Arch Gen Psychiatry 1995; 52: 1048-1060.

68 McCutcheon VV, Sartor CE, Pommer NE, Bucholz KK, Nelson EC, Madden PAF et al. Age at trauma exposure and PTSD risk in a young adult female sample. J Trauma Stress 2010; 23: 811-814.

69 Macciocchi S, Seel RT, Warshowsky A, Thompson N, Barlow K. Co-occurring traumatic brain injury and acute spinal cord injury rehabilitation outcomes. Arch Phys Med Rehabil 2012; 93: 1788-1794.

70 Hagen EM. Acute complications of spinal cord injuries. World J Orthop 2015; 6: 17-23.

71 O'Donnell ML, Creamer M, Holmes AC, Ellen S, McFarlane AC Judson $R$ et al. Posttraumatic stress disorder after injury: does admission to intensive care unit increase risk? J Trauma 2010; 69: 627-632.

72 Jackson JC, Jutte JE, Hunter $\mathrm{CH}$, Ciccolella $\mathrm{N}$, Warrington $\mathrm{H}$, Sevin $\mathrm{C}$ et al. Posttraumatic stress disorder (PTSD) after critical illness: a conceptual review of distinct clinical issues and their implications. Rehabil Psychol 2016; 61: 132-140.

73 McGiffin JN, Galatzer-Levy IR, Bonanno GA. Is the intensive care unit traumatic? What we know and don't know about the intensive care unit and posttraumatic stress responses. Rehabil Psychol 2016; 61: 120-131.

74 Kennedy P, Lowe R, Grey N, Short E. Traumatic spinal cord injury and psychological impact: a cross-sectional analysis of coping strategies. Br J Clin Psychol 1995; 34: 627-639.

75 Wade SL, Borawski EA, Taylor HG, Drotar D, Yeates KO, Stancin T. The relationship of caregiver coping to family outcomes during the initial year following pediatric traumatic injury. J Consult Clin Psychol 2001; 69: 406-415.

76 American Psychiatric Association. Diagnostic and Statistical Manual of Mental Disorders: DSM-5. American Psychiatric Association: Washington, USA, 2013.

77 Janoff-Bulman R. Shattered Assumptions: Towards a New Psychology of Trauma. Free Press: New York, USA, 1992

78 Sharp TJ, Harvey AG. Chronic pain and posttraumatic stress disorder: mutual maintenance? Clin Psychol Rev 2001; 21: 857-877.

79 Kisala PA, Victorson D, Pace N, Heinemann AW, Choi SW, Tulsky DS. Measuring psychological trauma after spinal cord injury: development and psychometric characteristics of the SCI-QOL Psychological Trauma item bank and short form. J Spinal Cord Med 2015; 38: 326-334.

80 Clarke DE, Kuhl EA. DSM- 5 cross-cutting symptom measures: a step towards the future of psychiatric care? World Psychiatry 2014; 13: 314-316.

81 Foa EB, Keane TM, Friedman MJ, Cohen JA. Effective Treatments for PTSD: Practice Guidelines from the International Society for Traumatic Stress Studies, 2nd edn. Guilford Press: New York, USA, 2008.

82 Kennedy P, Duff J, Evans M, Beedie A. Coping effectiveness training reduces depression and anxiety following traumatic spinal cord injuries. $\mathrm{Br} J$ Clin Psychol 2003; 42(Pt 1): 41-52

83 Mehta S, Orenczuk S, Hansen KT, Aubut J-AL, Hitzig SL, Legassic M et al. An evidence-based review of the effectiveness of cognitive behavioral therapy for psychosocial issues post-spinal cord injury. Rehabil Psychol 2011; 56: 15-25.

84 Huston T, Gassaway J, Wilson C, Gordon S, Koval J, Schwebel A. Psychology treatment time during inpatient spinal cord injury rehabilitation. J Spinal Cord Med 2011; 34: 196-204.

85 Kraft R, Dorstyn DS. Psychosocial correlates of depression following spinal injury: a systematic review. J Spinal Cord Med 2015; 38: 571-583.

86 Del Vecchio N, Elwy AR, Smith E, Bottonari KA, Eisen SV. Enhancing self-report assessment of PTSD: development of an item bank. J Trauma Stress 2011; 24: 191-199.

87 Liu S, Wang L, Cao C, Zhang J. The factor structure of posttraumatic stress disorder symptoms in patients with traumatic spinal cord injuries. Arch Psychiatr Nurs 2014; 28: 301-304.

88 Hinton DE, Lewis-Fernández R. The cross-cultural validity of post-traumatic stress disorder: implications for DSM-5. Depress Anxiety 2010; 28: 783-801.

89 DiGangi JA, Gomez D, Mendoza L, Jason LA, Keys CB, Koenen KC. Pretrauma risk factors for posttraumatic stress disorder: a systematic review of the literature. Clin Psychol Rev 2013; 33: 728-744.

90 Lammertse DP. Clinical trials in spinal cord injury: lessons learned on the path to translation. The 2011 International Spinal Cord Society Sir Ludwig Guttmann Lecture. Spinal Cord 2013; 51: 2-9. 


\section{APPENDIX}

Logic grids and boolean operators for electronic database searches

\section{PTSD}

CINAHL Stress Disorders, Post-Traumatic[mh] OR PTSD[tw] OR Post-Traumatic stress disorder[tw] OR Post-traumatic stress[tw] OR Post-traumatic stress[tw] OR post-traumatic psychos*[tw] OR post-traumatic syndrome[tw] OR post-traumatic neuros*[tw] OR post-traumatic psychos*[tw] OR post-traumatic [tw] OR spinal cord fracture*[tw] OR tetrapleg*[tiab] OR quadripleg*[tiab] OR syndrome[tw] OR post-traumatic neuros*[tw] OR traumatic stress[tiab] OR war parapleg*[tiab] neuros*[tiab] OR combat stress*[tiab] OR combat neuros*[tiab] OR shell shock [tiab] MH 'Stress disorders, post-traumatic' OR TX ‘stress disorders, post-traumatic' $M H$ ‘spinal cord injuries' OR MH ‘spinal injuries' OR TX 'spinal cord injur*' OR
OR TX PTSD OR TX ‘post-traumatic stress' OR TX 'post-traumatic stress' OR TI TX 'spinal fracture*' OR TX 'spine injur*' OR TX 'spinal cord trauma*' OR TX 'traumatic stress' OR AB 'traumatic stress' OR TX 'post-traumatic psychos*' OR 'spinal trauma*' OR TX 'spine trauma' OR TX 'spine fracture*' OR TX 'spinal TX 'post-traumatic syndrome' OR TX 'post-traumatic neuros' OR TX 'post-traumatic psychos' OR TX 'post-traumatic syndrome' OR TX 'post-traumatic cord fracture*' TI parapleg* OR AB parapleg* OR TI quadripleg* OR AB neuros*'

OR TI 'war neuros*' OR AB 'war neuros*' OR TI 'combat stress' OR AB 'combat stress' OR TI 'combat neuros*' OR AB 'combat neuros*' OR TI 'shell shock' OR $A B$ 'shell shock'

PSYCINFO (post-traumatic stress disorder or traumatic neurosis or stress reactions).sh. or PTSD.tw. or post-traumatic stress.tw. or post-traumatic stress.tw. or post-traumatic stress.tw. or post-traumatic psychos*.tw. or post-traumatic syndrome.tw. or post-traumatic neuros*.tw. or post-traumatic psychos*.tw. or post-traumatic syndrome.tw. or post-traumatic neuros* ${ }^{*}$.tw. or traumatic stress. ti,ab. or war neuros*.ti,ab. or combat stress.ti,ab. or combat neuros*.ti,ab. or shell shock.ti,ab.

PUBMED 'post-traumatic stress disorder'/syn OR 'post-traumatic stress' OR 'post-traumatic stress' OR 'combat neurosis' OR 'combat neuroses' OR 'shell shock' OR PTSD

SCOPUS ((ALL ('post-traumatic stress disorder' OR 'post-traumatic stress' OR 'post-traumatic stress' OR 'post-traumatic stress') OR TITLE-ABS-KEY('traumatic stress' OR 'war neuros*' OR 'combat stress' OR 'combat neuros*' OR 'shell shock' OR 'post-traumatic psychos*' OR 'post-traumatic syndrome' OR 'posttraumatic neuros' OR 'post-traumatic psychos' OR 'post-traumatic syndrome' OR 'post-traumatic neuros"' OR 'combat stress' OR 'traumatic neurosis' OR (PTSD')))

WEB OF TS = ('post-traumatic stress disorder' OR 'combat stress' OR 'traumatic neuSCIENCE rosis' OR 'PTSD' OR 'post-traumatic stress' OR 'post-traumatic stress' OR 'post-traumatic stress' OR 'traumatic stress' OR 'war neuros*' OR 'combat stress' OR 'combat neuros*' OR 'shell shock' OR 'post-traumatic psychos*' 'post-traumatic syndrome' OR 'post-traumatic neuros' OR 'post-traumatic psychos' OR 'post-traumatic syndrome' OR 'post-traumatic neuros ${ }^{* \prime}$ )

spinal cord injuries.sh OR spinal cord injur*.mp OR spine injur*.mp OR spinal injur*.mp OR spinal cord trauma*.mp OR spinal trauma*.mp OR spine trauma*.mp OR spinal fracture*.ti,ab OR spine fracture*.ti,ab OR spinal cord fracture*.tw OR parapleg*.ti,ab OR quadripleg*.ti,ab OR tetrapleg*.ti,ab

(n)

ssin

'spinal cord injury'/syn OR 'spine injury'/syn OR 'spine injury' OR 'spine injuries' OR quadripleg*:ab,ti OR 'tetrapleg*':ab,ti OR parapleg*:ab,ti ‘spinal cord traumas':ab,ti OR 'spinal traumas':ti,ab 'spine traumas':ti,ab OR 'spinal cord fracture':ti,ab OR 'spinal cord fractures':ti,ab OR 'spinal fracture':ti,ab OR 'spinal fractures':ti,ab OR 'spine fracture':ti,ab OR 'spine fractures':ti,ab

TITLE-ABS-KEY ('spinal cord injuries' OR 'spinal cord injur*' OR 'spine injur*' $O R$ 'spinal injur*' $O R$ 'spinal cord trauma*' $O R$ 'spinal fracture*' $O R$ 'spinal cord trauma*' OR 'spinal trauma*' OR 'spine trauma' OR 'spine fracture*' OR 'spinal cord fracture*' OR 'parapleg*' OR quadripleg* OR tetrapleg*)

TS = ('spinal cord injuries' OR 'spinal cord injur' OR 'spine injur*' OR 'spinal injur*'OR 'spinal cord trauma*' OR 'spinal trauma*' OR 'spine trauma' OR 'spinal fracture*' $O R$ 'spine fracture*' $O R$ 'spinal cord fracture*' OR 'parapleg*' OR OR quadripleg* OR tetrapleg*) 